\title{
A novel DEMATEL theory based on Liu's polytomous ordering theory
}

\author{
Hsiang-Chuan Liư ${ }^{1}$, Yih-Chang $\mathrm{Ou}^{2}$, Ben-Chang Shia ${ }^{3}$ and Hsien-Chang Tsai ${ }^{4, a}$ \\ ${ }^{1}$ Department of Informatics and Biomedical Engineering, Asia University, Taichung, Taiwan \\ ${ }^{2}$ Department of Finance, Ling Tung University, Taichung, Taiwan \\ ${ }^{3}$ Big Data Research Center \& School of Management School of Health Care Administration, Taipei Medical University, Taipei, Taiwan \\ ${ }^{4}$ Department of Biology, National Changhua University of Education, Changhua, Taiwan
}

\begin{abstract}
The most important issue in DEMATEL theory is how to obtain a reliable initial direct relation matrix with order $\mathrm{n}$, the traditional theory obtains it by using the pair-wise comparison method, in which, each respondent must answer $n(n-1)$ times pair-wise comparisons of all of the direct influences, if $\mathrm{n}$ is a large number, the work of pair-wise comparing is becoming hard, time-consuming, and unreliable. In this paper, for overcoming above drawbacks, we replace the pair-wise comparison method with Liu's ordering theory to find the initial direct relation matrix. This new method without pair-wise comparing can be used for any order $n$, a simple example was also provided in this paper to illustrate the advantages of the proposed theory.
\end{abstract}

\section{Introduction}

Decision Making Trial and Evaluation Laboratory (DEMATEL) was developed by Gabus and Fontela [1], Fontela and Gabus [2], and it can be used to resolve complex and difficult problems in the world, up to now, it has been widely used as one of the best tools to solve the cause and effect relationship among the evaluation factors [3-8].

\section{The Traditional DEMATEL}

The procedure of the traditional DEMATEL method is briefly introduced below:

Step 1: Calculate the initial direct relation matrix $Q$

If $\mathrm{n}$ factors are asked by $\mathrm{N}$ experts to evaluate the degree of direct influence between two factors based on pair-wise comparison.

The degree to which the expert e perceived factor $\mathrm{i}$ affects on factor $\mathrm{j}$ is denoted as

$$
q_{i j}^{(e)}, e=1,2, . ., N, q_{i j}^{(e)} \in\{0,1,2,3,4\}, i, j=1,2, \cdots, n
$$

For each expert e, an individual direct relation matrix is constructed as

$$
Q_{e}=\left[q_{i j}^{(e)}\right]_{n \times n}, e=1,2, . ., N, q_{i i}^{(e)}=0, i=1,2, \ldots, n
$$

and we can obtain their average direct relation matrix, called the initial direct relation matrix Q;

$$
Q=\left[q_{i j}\right]_{n \times n}=\frac{1}{N} \sum_{e=1}^{N} Q_{e}, q_{i j}=\frac{1}{N} \sum_{e=1}^{N} q_{i j}^{(e)}, i, j=1,2, \cdots, n
$$

Step 2: Calculate the direct relation matrix A

$$
A=\left[a_{i j}\right]_{n \times n}=\lambda^{-1} Q, \lambda=\max _{1 \leq i, j \leq n}\left\{\sum_{j=1}^{n} q_{i j}, \sum_{i=1}^{n} q_{i j}\right\}
$$

where

$$
\begin{gathered}
a_{i i}=0, i=1,2, \ldots, n, \quad 0 \leq a_{i j} \leq 1, i \neq j, i, j=1,2, \ldots, n \\
\text { and } \quad 0 \leq \sum_{i=1}^{n} a_{i j}, \sum_{j=1}^{n} a_{i j} \leq 1, i, j=1,2, \ldots, n
\end{gathered}
$$

Step 3: Calculate the indirect relation matrix B and the total relation matrix $\mathrm{T}$

Based on Markov chain theory, we have

$$
\begin{gathered}
\lim _{k \rightarrow \infty} A^{k}=0_{n \times n} \\
B=\left[b_{i j}\right]_{n \times n}=\lim _{k \rightarrow \infty}\left[A^{2}+A^{3}+\ldots+A^{k}\right]=A^{2}(I-A)^{-1} \\
T=\left[t_{i j}\right]_{n \times n}=A+B=\left[\left(a_{i j}+b_{i j}\right)\right]_{n \times n}
\end{gathered}
$$

Step 4: Calculate the relation degree and prominence degree of each factor

$$
r_{i}=\sum_{j=1}^{n} t_{i j}, \quad c_{i}=\sum_{k=1}^{n} t_{k i}, \quad i=1,2, \ldots, n
$$

The value of $r_{i}$ indicates the total dispatch both directly and indirectly effects, that factor $i$ has on the other factors, and the value of $c_{i}$ indicates the total receive both directly and indirectly effects, that factor $\mathrm{i}$ has on the other factors.

The relation degree of factor $\mathrm{i}$ is denoted as

$$
x_{i}=r_{i}-c_{i}, \quad i=1,2, \ldots, n
$$

The prominence degree of factor $\mathrm{i}$ is denoted as

$$
y_{i}=r_{i}+c_{i}, \quad i=1,2, \ldots, n
$$

relation -prominence matrix;

$$
\left(x_{i}, y_{i}\right)_{i=1}^{n}
$$

Step 5: Set the threshold value $(\alpha)$

For eliminating some minor effects elements in matrix $\mathrm{T}$ to find the impact-relations map, Yang et al.[5] suggest their threshold value below;

\footnotetext{
$\overline{{ }^{a} \text { Corresponding author: bihft@cc.ncue.edu.tw }}$
} 


$$
\alpha_{Y}=\frac{1}{n^{2}} \sum_{i=1}^{n} \sum_{j=1}^{n} t_{i j}
$$

Lin and Tzeng [6] suggested a more information threshold value, $\alpha_{M}$, based on their maximum mean deentropy (MMDE) algorithm.

Step 6: Build a cause and effect relationship diagram

If $t_{i j}>\alpha_{Y}$, or $t_{j i}>\alpha_{Y}$, then factor $\mathrm{i}$ is a net dispatch node of factor $\mathrm{j}$, and factor $\mathrm{j}$ is a net receive node of factor $\mathrm{i}$, respectively, and denoted as

$$
\left(x_{i}, y_{i}\right) \rightarrow\left(x_{j}, y_{j}\right), \text { or }\left(x_{i}, y_{i}\right) \leftarrow\left(x_{j}, y_{j}\right)
$$

The graph of $\left(x_{i}, y_{i}\right)_{i=1}^{n}$ including the net direct edges can present a cause and effect relationship diagram.

\section{New DEMATEL theory based on Liu's polytomous ordering theory}

\subsection{Liu's polytomous ordering theory}

If $\mathrm{n}$ factors are asked by $\mathrm{N}$ experts to evaluate the degree of direct influence of each factor i to the rest, the degree to which the expert e perceived factor $i$ affects on the rest is denoted as

Let $M=\{0,1,2, \ldots,(m-1)\}$ and the data matrix is denoted below;

$$
D=\left[d_{i e}\right]_{n \times N}, d_{i e} \in M, i=1,2, \ldots, n, e=1,2, \ldots, N
$$

Let $S_{i}(x)=\left\{d_{i e} \mid d_{i e}=x, e=1,2, . ., N\right\}, x \in M, i=1,2, \ldots, n$ (15)

$$
S_{i j}(x, y)=\left\{\left(d_{i e}, d_{j e}\right) \mid\left(d_{i e}, d_{j e}\right)=(x, y), e=1,2, . ., N\right\}
$$

where $i, j=1,2, \ldots, n, \quad x, y \in M$,

then the probabilities of impact degree of factor are defined below;

$$
\begin{gathered}
P_{i}(x)=\frac{\left|S_{i}(x)\right|}{N}, P_{j}(y)=\frac{\left|S_{j}(y)\right|}{N}, P_{i j}(x, y)=\frac{\left|S_{i j}(x, y)\right|}{N} \\
\text { Let } \quad P_{i}=\sum_{x=0}^{m-1} P_{i}(x), \quad P_{j}=\sum_{y=0}^{m-1} P_{j}(y),
\end{gathered}
$$

$P_{i}>P_{j}$ means that the degree of direct influence of factor $\mathrm{i}$ to others is greater than which of factor $\mathrm{j}$.

Liu's ordering coefficient from factor i to factor i [8] is defined below;

$$
\begin{gathered}
r_{i j}^{(L O T)}=1-V_{i j} \\
\text { where } P_{i j}(x>y)=\frac{1}{(m-1)}[x-y]^{+} P_{i j}(x, y) \\
{[z]^{+}= \begin{cases}z & \text { if } z>0 \\
0 & \text { if } z \leq 0\end{cases} }
\end{gathered}
$$

In (20) and (21), $P_{i j}(x>y)$ represents the impact degree of factor $\mathrm{i}$ is greater than which of factor $\mathrm{j}$, hence $V_{i j}$ is just the violated probability.

For all experts. Liu's ordering coefficient from factor $\mathrm{i}$ to factor $\mathrm{j}, r_{i j}^{(L O T)}$, represents that the all probable probability minas the violated probability, it is reasonable and well defined.

\subsection{Example for Liu's polytomous ordering theory}

Example 1. Suppose the joint probability and marginal probability of the impact degree $\mathrm{x}$ of factor 1 and the impact degree y of factor 2 are listed in Table 1. below, from formula (20), we can get $V_{12}=0.3050$, $V_{21}=0.2700$, then using formula (19), we have $r_{12}^{(\text {LOT })}=0.6950$ and $r_{21}^{(\text {LOT })}=0.7300$.

Similarly, we can obtain the values of all Liu's ordering coefficients;

$r_{i j}^{(L O T)}, i \neq j, i, j=1,2, \ldots, n$

Table 1 . The probabilities of $P_{12}(x, y)$

\begin{tabular}{|c|c|c|c|c|c|c|}
\hline$P_{12}(x, y)$ & $y=0$ & $y=1$ & $y=2$ & $y=3$ & $y=4$ & $P_{1}(x)$ \\
\hline$x=0$ & 0.03 & 0.05 & 0.06 & 0.02 & 0.01 & 0.17 \\
\hline$x=1$ & 0.05 & 0.04 & 0.05 & 0.04 & 0.02 & 0.20 \\
\hline$x=2$ & 0.05 & 0.05 & 0.13 & 0.03 & 0.01 & 0.27 \\
\hline$x=3$ & 0.05 & 0.03 & 0.04 & 0.11 & 0.03 & 0.26 \\
\hline$x=4$ & 0.02 & 0.01 & 0.02 & 0.01 & 0.04 & 0.10 \\
\hline$P_{2}(y)$ & 0.20 & 0.18 & 0.30 & 0.21 & 0.11 & 1.00 \\
\hline
\end{tabular}

\subsection{New DEMATEL theory based on Liu's ordering theory}

The procedure of the new DEMATEL theory based on Liu's ordering theory is briefly described below:

Step 1: Calculate the data matrix D

If $\mathrm{n}$ factors are asked by $\mathrm{N}$ experts to evaluate the degree of direct influence of each factor i to the rest, according to equation (14) to calculate the data matrix;

$$
D=\left[d_{i e}\right]_{n \times N}, d_{i e} \in\{0,1,2,3,4\} .
$$

Step 2: Calculate the probabilities of impact degree of each factor

According to equations (14)-(18) to calculate the probabilities; $P_{i}(x), P_{j}(y), P_{i j}(x, y)$.

Step 3: Calculate the Liu's ordering coefficient for each order-pair of all factors

According to equations (19)-(22) to calculate Liu's ordering coefficient from factor i to factor $\mathrm{j}$;

$$
r_{i j}^{(L O T)}, \quad i, j=1,2, \ldots, n
$$

Step 4: Calculate the initial direct relation matrix $\mathrm{Q}$ 


$$
\begin{array}{r}
Q=\left[q_{i j}\right]_{n \times n}, q_{i i}=0, i=1,2, \ldots, n, \\
q_{i j}=r_{i j}^{(\text {LOT })}, i \neq j, i, j=1,2, \ldots, n
\end{array}
$$

Step 5: Calculate the direct relation matrix A

According to equations (24) to calculate A

$$
A=\left[a_{i j}\right]_{n \times n}=\lambda^{-1} Q, \lambda=\max _{1 \leq i, j \leq n}\left\{\sum_{j=1}^{n} q_{i j}, \sum_{i=1}^{n} q_{i j}\right\}
$$

The rest steps are the same as the traditional DEMATEL theory.

\subsection{Example for New DEMATEL theory}

Example 2 Suppose 4 factors are asked by 10 experts to evaluate the degree of direct influence of each factor $i$ to the rest, according to the step 1, up to step 4 of the new method, we can obtain Liu's ordering coefficient of 4 factors and the initial direct relation matrix $Q$ can be obtained, each expert does not need repeat the hard work by pair-wise comparison 4(4-1) times.

Step 1-Step 4.: Calculate Liu's ordering coefficient of 4 factors and the initial direct relation matrix Q According to equations (19)-(22), we can obtain Liu's ordering coefficient matrix of 4 factors and the initial direct relation matrix $\mathrm{Q}$ below;

$$
\begin{aligned}
& Q=\left[q_{i j}\right]_{4 \times 4}=\left[r_{i j}^{(L O T)}\right]_{4 \times 4} \\
& =\left[\begin{array}{cccc}
0 & 0.72 & 0.64 & 0.64 \\
0.64 & 0 & 0.68 & 0.60 \\
0.68 & 0.60 & 0 & 0.60 \\
0.56 & 0.56 & 0.60 & 0
\end{array}\right]
\end{aligned}
$$

Step 5: Calculate the direct relation matrix A According to equations (4), we have

$$
\begin{aligned}
\lambda=\max _{1 \leq i, j \leq n}\left\{\sum_{j=1}^{n} q_{i j}, \sum_{i=1}^{n} q_{i j}\right\}=2 \\
A=\left[a_{i j}\right]_{4 \times 4}=\lambda^{-1} Q \\
=\left[\begin{array}{cccc}
0 & 0.36 & 0.32 & 0.32 \\
0.32 & 0 & 0.34 & 0.30 \\
0.34 & 0.30 & 0 & 0.30 \\
0.28 & 0.28 & 0.30 & 0
\end{array}\right]
\end{aligned}
$$

Step 6: Calculate the indirect relation matrix $\mathrm{B}$

$$
\begin{aligned}
& B=\left[b_{i j}\right]_{4 \times 4}=A^{2}(I-A)^{-1} \\
& =\left[\begin{array}{llll}
3.9448 & 3.8483 & 3.9290 & 3.7995 \\
3.7483 & 3.8237 & 3.7991 & 3.6907 \\
3.6807 & 3.6963 & 3.8253 & 3.6331 \\
3.4499 & 3.4478 & 3.4963 & 3.4508
\end{array}\right]
\end{aligned}
$$

Step 7: Calculate the total relation matrix $\mathrm{T}$

$$
\begin{aligned}
T & =\left[t_{i j}\right]_{4 \times 4}=A+B=\left[\left(a_{i j}+b_{i j}\right)\right]_{4 \times 4} \\
& =\left[\begin{array}{llll}
3.9448 & 4.2083 & 4.2490 & 4.1195 \\
4.0683 & 3.8237 & 4.1391 & 3.9907 \\
4.0207 & 3.9963 & 3.8253 & 3.9331 \\
3.7299 & 3.7278 & 3.7963 & 3.4508
\end{array}\right]
\end{aligned}
$$

Step 8: Calculate the relation degree and prominence degree of each factor and relation prominence matrix;

$$
\begin{array}{r}
x_{i}=r_{i}-c_{i}=\sum_{j=1}^{4} t_{i j}-\sum_{k=1}^{4} t_{k i}, \quad i=1,2, \ldots, n \\
y_{i}=r_{i}+c_{i}=\sum_{j=1}^{4} t_{i j}+\sum_{k=1}^{4} t_{k i}, \quad i=1,2, \ldots, n \\
\left(x_{i}, y_{i}\right)_{i=1}^{4}=\left[\begin{array}{l}
\left(x_{1}, y_{1}\right) \\
\left(x_{2}, y_{2}\right) \\
\left(x_{3}, y_{3}\right) \\
\left(x_{4}, y_{4}\right)
\end{array}\right]=\left[\begin{array}{ll}
(0.7579, & 32.2851) \\
(0.2658, & 31.7781) \\
(-0.2344, & 31.7850) \\
(-0.7893, & 30.1989)
\end{array}\right]
\end{array}
$$

Step 9: Set the threshold value and find the significant values using Liu's threshold value below;

$$
\begin{gathered}
\alpha_{L}=\max \left\{\alpha_{Y}, \max _{i=1,2, \ldots n} t_{i i}\right\}=3.9448 \\
\text { Where } \alpha_{Y}=\frac{1}{n^{2}} \sum_{i=1}^{n} \sum_{j=1}^{n} t_{i j}=3.9390
\end{gathered}
$$

If $t_{i j}>\alpha_{Y}=3.9390$, then $t_{i j}$ is a significant value, denoted $t_{i j}^{*}, \quad i, j=1,2, \ldots, n$,

$$
T^{*}=\left[\begin{array}{llll}
3.9448 & 4.2083^{*} & 4.2490^{*} & 4.1195^{*} \\
4.0683^{*} & 3.8237 & 4.1391^{*} & 3.9907^{*} \\
4.0207^{*} & 3.9963^{*} & 3.8253 & 3.9331 \\
3.7299 & 3.7278 & 3.7963 & 3.4508
\end{array}\right]
$$

Step 10: Build a cause and effect relationship diagram according to the reduced $T^{*}$ matrix

The formal definition of the reduced $T^{*}$ matrix is defined as follows;

If $t_{i j}>\alpha_{Y}$, then factor $\mathrm{i}$ is significantly a dispatch of factor $\mathrm{j}$, denoted as $x_{i} \rightarrow x_{j}, \quad i, j=1,2, \ldots, n$, the reduced $T^{*}$ matrix is denoted as;

$$
T_{r e d}^{*}=\left[z_{i j}\right]_{n \times n}, \text {, where } z_{i j}= \begin{cases}1 & \text { if } x_{i} \rightarrow x_{j} \\ 0 & \text { if } x_{i} \nrightarrow x_{j}\end{cases}
$$

For our example, we can obtain the reduced $T^{*}$ matrix below;

$$
T_{\text {red }}^{*}=\left[z_{i j}\right]_{4 \times 4}=\left[\begin{array}{cccc}
0 & 1 & 1 & 1 \\
1 & 0 & 1 & 1 \\
1 & 1 & 0 & 0 \\
0 & 0 & 0 & 0
\end{array}\right]
$$

and then, the cause and effect relationship diagram can easily be constructed. 


\section{Conclusion}

In this paper, we pointed out that each respondent answering the questionnaire of the traditional DEMATEL by pair-wise comparison is a hard, timeconsuming, and unreliable work, if the number of factors is large. For overcoming this drawback, we replaced the pair-wise comparison method with Liu's ordering theory to find a more reliable initial direct relation matrix without pair-wise comparison, and this new method can be used for any number of factors. A simple example was also provided to illustrate the advantages of the proposed theory.

This paper is partially supported by the National Science Council grant (NSC 100-2511-S-468 -001).

\section{References}

1. A. Gabus, E. Fontela, Perceptions of the World Problematique: Communication Procedure, Communicating with those Bearing Collective Responsibility (Switzerland Geneva, 1973)

2. E. Fontela, A. Gabus, The DEMATEL observer, (Switzerland Geneva, 1976)

3. G.H. Tzeng, C.H. Chiang, C.W. Li, Expert. Syst. Appl 32, 4 (2007)

4. J.H. Liou, L. Yen, G.H. Tzeng, J. Air. Transp. Manag 14, 1 (2008)

5. Y.P.O. Yang, H.M. Shieh, J.D. Leu, G.H. Tzeng, Int. J. Oper. Res 5, 3 (2008)

6. C.L. Lin, G.H. Tzeng, Expert. Syst. Appl 36, 6 (2009)

7. C.W. Li, G.H. Tzeng, Expert. Syst. Appl 36, 6 (2009)

8. H.C. Liu, W.S. Chen, C.M. Chiang, B.C. Shia, J.M. Ju, ICIC Express Lett Part B Appl 6, 2 (2015) 